\title{
Both attention and prediction are necessary for adaptive neuronal tuning in sensory processing
}

\author{
Yi-Fang Hsu ${ }^{1,2}$ *, Jarmo A. Hämäläinen ${ }^{3}$ and Florian Waszak ${ }^{1,2}$ \\ ' Université Paris Descartes, Sorbonne Paris Cité, Paris, France \\ ${ }^{2}$ CNRS, Laboratoire Psychologie de la Perception, UMR 8242, Paris, France \\ ${ }^{3}$ Department of Psychology, University of Jyväskylä, Jyväskylä, Finland
}

\section{Edited by:}

Harriet Brown, University College London, UK

\section{Reviewed by:}

Ana Todorovic, Radboud University Nijmegen, Netherlands

Ryszard Auksztulewicz, University

College London, UK

\section{*Correspondence:}

Yi-Fang Hsu, Université Paris

Descartes, Sorbonne Paris Cité and CNRS, Laboratoire sychologie de la Perception, UMR 8242, 45 Rue des Saints-Pères, 75006 Paris, France e-mail:yi-fang.hsu@cantab.net
The brain as a proactive system processes sensory information under the top-down influence of attention and prediction. However, the relation between attention and prediction remains undetermined given the conflation of these two mechanisms in the literature. To evaluate whether attention and prediction are dependent of each other, and if so, how these two top-down mechanisms may interact in sensory processing, we orthogonally manipulated attention and prediction in a target detection task. Participants were instructed to pay attention to one of two interleaved stimulus streams of predictable/unpredictable tone frequency. We found that attention and prediction interacted on the amplitude of the N1 ERP component. The N1 amplitude in the attended/predictable condition was larger than that in any of the other conditions. Dipole source localization analysis showed that the effect came from the activation in bilateral auditory areas. No significant effect was found in the P2 time window. Our results suggest that attention and prediction are dependent of each other. While attention might determine the overall cortical responsiveness to stimuli when prediction is involved, prediction might provide an anchor for the modulation of the synaptic input strengths which needs to be operated on the basis of attention.

Keywords: attention, prediction, sensory processing, electroencephalography, event-related potentials

\section{INTRODUCTION}

Recent theories of sensory processing consider the brain as a proactive system which adapts quickly to the environment. Neurons in the sensory cortices can undergo short-term, task-dependent, and context-specific changes in receptive field properties when attention and prediction are involved (Fritz etal., 2003, 2007, 2008). Such adaptive plasticity driven by attention and prediction can be the underlying mechanism for the optimization ofperception.

Attention is suggested to have a global effect on perception at an early stage of sensory processing. Electroencephalography (EEG) studies revealed the neuronal consequences of attention on event-related potentials (ERPs), particularly the enhancement of the N1 (Hillyard et al., 1973; Alcaini et al., 1994; Lange etal., 2003, 2006; Lange and Röder, 2006). This may result from changes in the selectivity of neurons in the sensory cortex (Chawla et al., 1999; Kastner et al., 1999; Ahveninen et al., 2006). Specifically, research showing that the auditory N1 response is modulated by task demands and notched-noise masking suggests that the spectrotemporal receptive fields of neurons are tuned according to attentional manipulations (Kauramäki et al., 2007), as attention excites neurons responsive to attended features and inhibits neurons responsive to unattended features (Fritz et al., 2003, 2007, 2008; Jääskeläinen et al., 2007). Neurocomputational studies demonstrated that attention may function via optimizing the synaptic gain to represent the precision of sensory information during hierarchical inference (Feldman and Friston, 2010).
Prediction, or the statistical regularity in the environment, is also suggested to modulate the early stage of sensory processing, albeit its effect on ERPs manifests as a suppression of the N1 (Lange, 2013). Prediction-related N1 suppression was demonstrated when participants had foreknowledge of the upcoming stimuli (Schafer and Marcus, 1973; Schafer et al., 1981; Lange, 2009; SanMiguel et al., 2013; Timm et al., 2013). The predictive coding model postulates that the prediction effect indexes the difference in neurocomputational demand for predictable/unpredictable information (Friston, 2005; Egner et al., 2010). Specifically, it indexes how much of the sensory input cannot be accounted for by the internal model. Moreover, prediction was reported to alter the contrast gain of sensory evidence accumulation (Melloni et al., 2011; Rohenkohl et al., 2012). Neurophysiologically, this is reflected in sharper sensory representations where the reduction of neuronal activity is smaller in neurons responsive to predictable features than in neurons responsive to unpredictable features (Kok et al., 2012a).

Despite their ERP effects being opposite, the relation of attention and prediction remains undetermined. This might be due to the conflation of these two mechanisms in the literature, where attention and prediction were often treated as the same concept (Corbetta and Shulman, 2002). However, attention and prediction can rely on orthogonal sources of information (Summerfield and Egner, 2013). While attention operates on the basis of motivational relevance, prediction operates on the basis of prior likelihood (Summerfield and Egner, 2009). It is possible that attention and prediction are two independent 
mechanisms which may have antagonistic (Summerfield and Egner, 2009) or additive (Timm et al., 2013) effects on neuronal signals for sensory processing. Alternatively, attention and prediction may be dependent of each other. One possibility is that one of the two mechanisms is necessary for the other to take effect, but not the other way round (Kok et al., 2012b). Another possibility is that such dependency is bidirectional, with both attention and prediction being necessary to modulate sensory processing.

To examine the relation between these two top-down mechanisms, we orthogonally manipulated attention and prediction in a target detection task. Participants were instructed to pay attention to one of two interleaved stimulus streams of predictable/unpredictable tone frequency. Using EEG, we quantified $\mathrm{N} 1$ and $\mathrm{P} 2$ as dependent variables given that the former is involved in auditory perception and the latter is suggested to reflect the comparison between the sensory input and the internal model (Evans and Federmeier, 2007; Costa-Faidella et al., 2011). The design allowed us to evaluate whether attention and prediction are dependent of each other, and if so, how these two top-down mechanisms may interact on sensory processing.

\section{MATERIALS AND METHODS PARTICIPANTS}

Sixteen healthy volunteers (average age 28; six males; all righthanded) with no history of neurological, psychiatric, or hearing impairments as indicated by self-report participated in the experiment. Participants gave written informed consent and were paid for participation. Ethical approval was granted by the Comité de Protection des Personnes (CPP) Ile de France II. The experiment conforms with The Code of Ethics of the World Medical Association (Declaration of Helsinki).

\section{STIMULI}

Sinusoidal tones with a loudness of 80 phons (i.e., $80 \mathrm{~dB}$ for tones of $1000 \mathrm{~Hz}$ ) were generated using Matlab. The duration of each tone was $50 \mathrm{~ms}$ (including $5 \mathrm{~ms}$ rise/fall times). The frequency of each tone was within the range of $261.626-493.883 \mathrm{~Hz}$ and 2093.000-3951.070 Hz, matching the absolute frequency of two sets of seven natural keys on a modern piano (low frequency set: C4 D4 E4 F4 G4 A4 B4; high frequency set: C7 D7 E7 F7 G7 A7 B7). Within each frequency set, the predictable/unpredictable stimulus streams were created. The predictable stimulus stream consisted of 400 pairs of tones. Each pair of tones was arranged in ascending order with the second tone being two natural keys higher than the first tone (e.g., C4-E4; G7-B7). The unpredictable stimulus stream consisted of 400 pairs of tones. Each pair of tones was arranged in random order with the second tone being any tone except the repetition of the first tone (e.g., C4-F4; G7F7). The tones in each condition were of equal variability in frequency.

The predictable/unpredictable stimulus streams from different frequency sets were interleaved to allow for the efficient manipulation of attention on the two stimulus sets. To counterbalance the mapping between predictable/unpredictable stimulus streams and high/low frequency sets, half of the participants were presented with a low-frequency predictable stimulus stream interleaved with a high-frequency unpredictable stimulus stream and half of the participants were presented with a high-frequency predictable stimulus stream interleaved with a low-frequency unpredictable stimulus stream. To counterbalance the sequential relation of the predictable/unpredictable stimulus streams, half of the blocks started with the predictable stimulus stream and half of the blocks started with the unpredictable stimulus stream. A stimulus onset asynchrony (SOA) of $500 \mathrm{~ms}$ was used (Figure 1). 10\% of the tones in one of the two stimulus

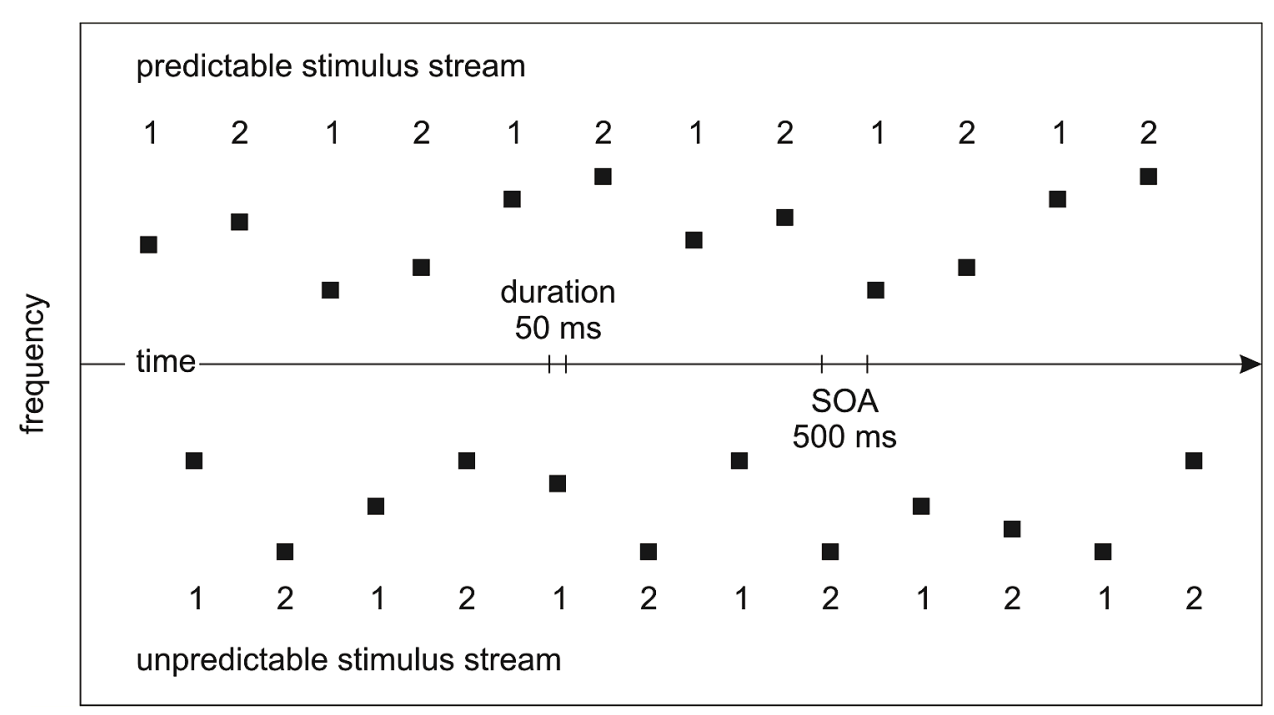

FIGURE 1 | Experimental design. The predictable/unpredictable stimulus streams from different frequency sets were interleaved. The numbers indicate the tones being the first/second tones in each pairs of tones.
Unaware of the manipulation of prediction on tone frequency, participants were instructed to pay attention to one of the stimulus streams in different blocks where tones of attenuated loudness may appear. 
streams served as targets attenuated in loudness by $20 \mathrm{~dB}$, independent of whether they were the first/second tones in each pair of tones. Stimulation was created individually for each participant.

\section{PROCEDURES}

Participants were presented with a total of eight blocks of 100 pairs of tones via headphones (Sennheiser PX200), with each block including 50 predictable pairs of tones and 50 unpredictable pairs of tones. Unaware of the manipulation of prediction, participants were instructed to pay attention to one of the stimulus streams (i.e., high/low frequency) in different blocks where tones of attenuated loudness may appear. To monitor whether participants followed the instructions correctly, participants were required to press a key when they detected a softer tone, which randomly occurred 10 times in each block. No practice session was provided. Block order was counterbalanced across participants. The experiment was administered conjointly with another EEG experiment which is to be reported elsewhere.

The stimuli of interest were the second tones in each pair of tones, which can be attended/predictable, attended/unpredictable, unattended/predictable, and unattended/unpredictable. Note that the manipulations of attention and prediction were both introduced on the basis of tone frequency. Moreover, all stimuli were presented binaurally. Therefore, there was no spatial effect in the current study.

\section{DATA RECORDING AND ANALYSIS \\ EEG recording and pre-processing}

EEG was recorded with 64 active electrodes (actiCAP, Brain Products $\mathrm{GmbH}$, Germany) conforming to the international 10-10 system. The sampling rate was $500 \mathrm{~Hz}$. No online/offline filter was used. The data was recomputed to average reference. Target stimuli and the first stimuli following target stimuli were removed. Epochs extended from -100 to $500 \mathrm{~ms}$ relative to stimulus onset, using a $100 \mathrm{~ms}$ pre-stimulus baseline. Ocular artifact correction was conducted with independent component analysis in EEGlab (Delorme and Makeig, 2004). Epochs containing voltage deviations exceeding $\pm 80 \mu \mathrm{V}$ relative to baseline at any of the electrodes were rejected. The trial numbers after artifact rejection in each condition are listed in Table $\mathbf{1 .}$

\section{ERP analysis}

ERP analysis was based on a temporal principal component analysis (PCA) in SPSS 20. The temporal PCA statistically decomposes the ERP waveforms into constituent building blocks, which

Table 1 | Mean and range of trial numbers after artifact rejection in each condition.

\begin{tabular}{lcccc}
\hline & $\begin{array}{c}\text { Attended } \\
\text { predictable }\end{array}$ & $\begin{array}{c}\text { Attended } \\
\text { unpredictable }\end{array}$ & $\begin{array}{c}\text { Unattended } \\
\text { predictable }\end{array}$ & $\begin{array}{c}\text { Unattended } \\
\text { unpredictable }\end{array}$ \\
\hline Mean & 175.38 & 171.38 & 171.81 & 173.50 \\
Range & $165-182$ & $141-187$ & $142-188$ & $161-181$
\end{tabular}

affords objective data-driven ERP component measures when compared to the conventional peak-picking methods (Kayser and Tenke, 2003; Dien et al., 2005; Kayser and Tenke, 2006; Dien, 2012). Moreover, it is not susceptible to the influences of highfrequency noises and low-frequency drifts in the data as the conventional peak-picking methods (Luck, 2005). Covariance matrix and Promax rotation were used. All components accounting for a total of $99 \%$ of the variance (maximum iterations for convergence $=500$ ) were included in the rotation (Promax Kappa $=4)$. The temporal decomposition provided a set of component loadings reflecting the contribution of each time point on the temporal principal components. The component loadings were used to derive component scores which represent the magnitude of neural activity within the time windows of interest. We identified $\mathrm{N} 1$ and P2 by selecting components showing typical N1 and P2 latency and topography. Electrodes showing the largest N1 component score (i.e., F2) and P2 component score (i.e., $\mathrm{Cz}$ ) were considered as representative electrodes for the components of interest. Therefore, their component scores were used as input for the 2 (attended/unattended) $\times 2$ (predictable/unpredictable) repeated measures analysis of variance (ANOVA).

\section{RESULTS \\ BEHAVIOURAL DATA}

Overall, participants' performance in the target detection task was at ceiling (Hit: mean $=0.96, \mathrm{SD}=0.03$; False alarm: mean $<0.01$, $\mathrm{SD}<0.01$; RT: mean $=539.34, \mathrm{SD}=62.21)$, confirming that participants followed the instructions correctly. The ceiling performance rendered it unlikely that participants' attention alternated between the two stimulus streams within blocks, which would have brought down participants' performance. There was no difference between participants' performance when they attended to predictable/unpredictable stimulus stream [Hit: $t(15)=0.30$, $p=0.77$; False alarm: $t(15)<0.01, p=1.00$; RT: $t(15)=0.32$, $p=0.75]$. The equivalent performance across blocks excluded the possibility that task difficulty may be a confounding factor in the current study.

\section{ERP DATA}

Figure 2 shows the grand average ERPs on nine representative electrodes (F3, Fz, F4, C3, Cz, C4, P3, Pz, P4). N1 with a frontocentral distribution and $\mathrm{P} 2$ with a central distribution are evident. Figure 3 shows the component loadings of 111 components in the temporal PCA. The temporal PCA yielded components clearly corresponding to N1 and P2 in the grand average ERPs. Figure 4 shows the $\mathrm{N} 1$ and P2 component scores on electrodes showing the largest responses (N1: F2; P2: Cz) and the topographical distribution of the components in each condition.

N1

The $2 \times 2$ repeated measures ANOVA showed a significant main effect of attention with attended stimuli triggering enhanced activity compared to unattended stimuli $[F(1,15)=6.50, p<0.05]$ and a marginally significant main effect of prediction with predictable stimuli triggering enhanced activity compared to unpredictable stimuli $[F(1,15)=3.40, p<0.10]$. More importantly, there was 

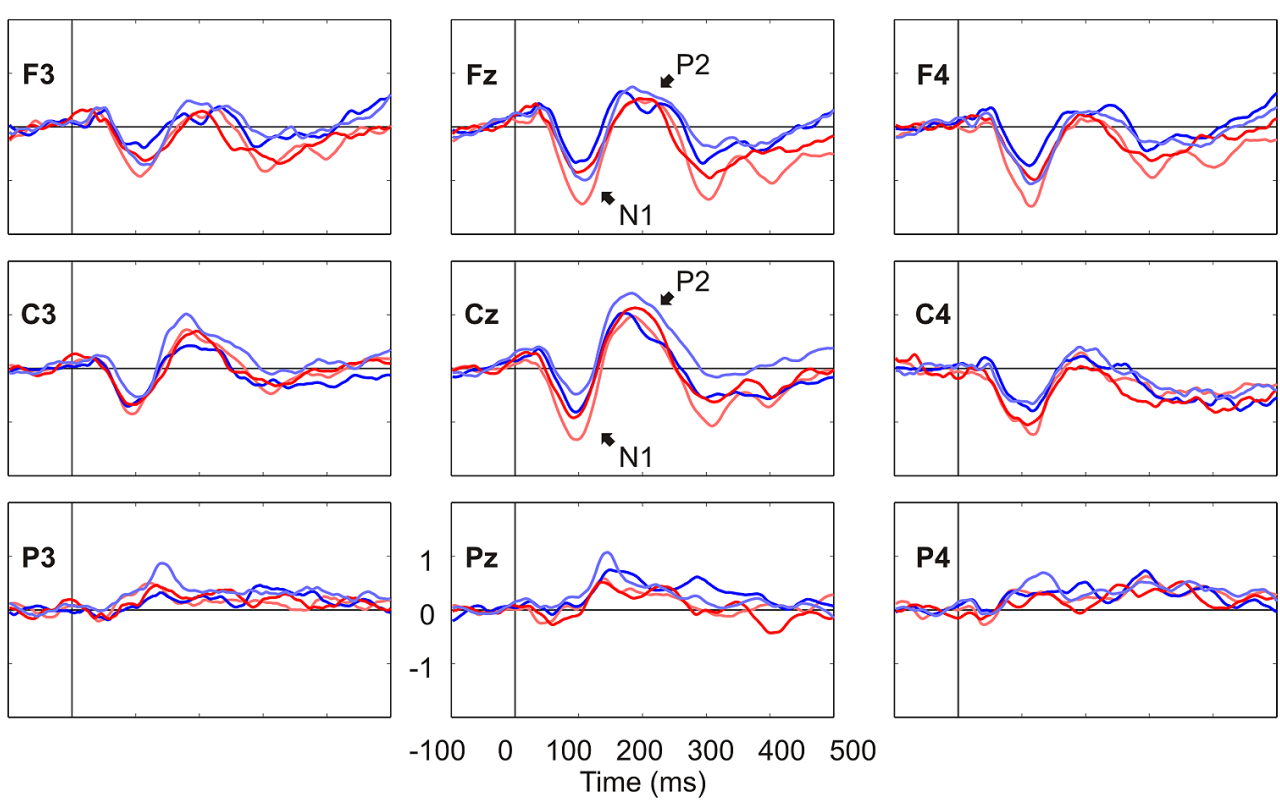

FIGURE 2 | Grand average ERPs on nine representative electrodes (F3, Fz, F4, C3, Cz, C4, P3, Pz, P4) lowpass filtered at $30 \mathrm{~Hz}$ for visual presentation purposes. $\mathrm{N} 1$ and $\mathrm{P} 2$ in the grand average ERPs are denoted.

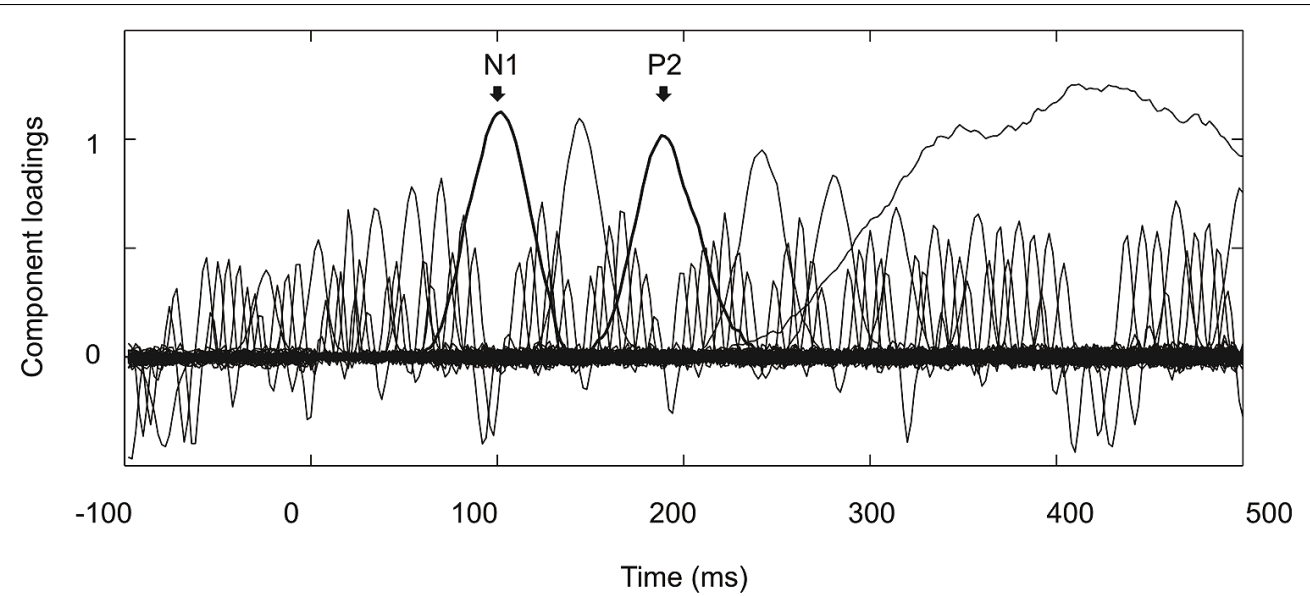

FIGURE 3 | Component loadings of 111 components in the temporal PCA. Components clearly corresponding to N1 and P2 in the grand average ERPs are marked with thick lines.

a significant attention $\times$ prediction interaction $[F(1,15)=4.61$, $p<0.05]$. Post hoc comparisons showed that the component score was larger for attended stimuli than for unattended stimuli in the predictable condition $[t(15)=-3.42, p<0.01]$ but not in the unpredictable condition $[t(15)=-0.60, p=0.56]$ and that the component score was larger for predictable stimuli than for unpredictable stimuli in the attended condition $[t(15)=-2.40, p<0.05]$ but not in the unattended condition $[t(15)=-0.63, p=0.54]$. Moreover, the component score for attended/predictable stimuli was larger than that for unattended/unpredictable stimuli $[t(15)=-2.44, p<0.05]$. In other words, the component score in the attended/predictable condition was larger than that in any of the other conditions. To examine the neural origin of the effects of attention and prediction, the component scores on all electrodes for N1 were further imported into BESA Research 6.0 for dipole source 


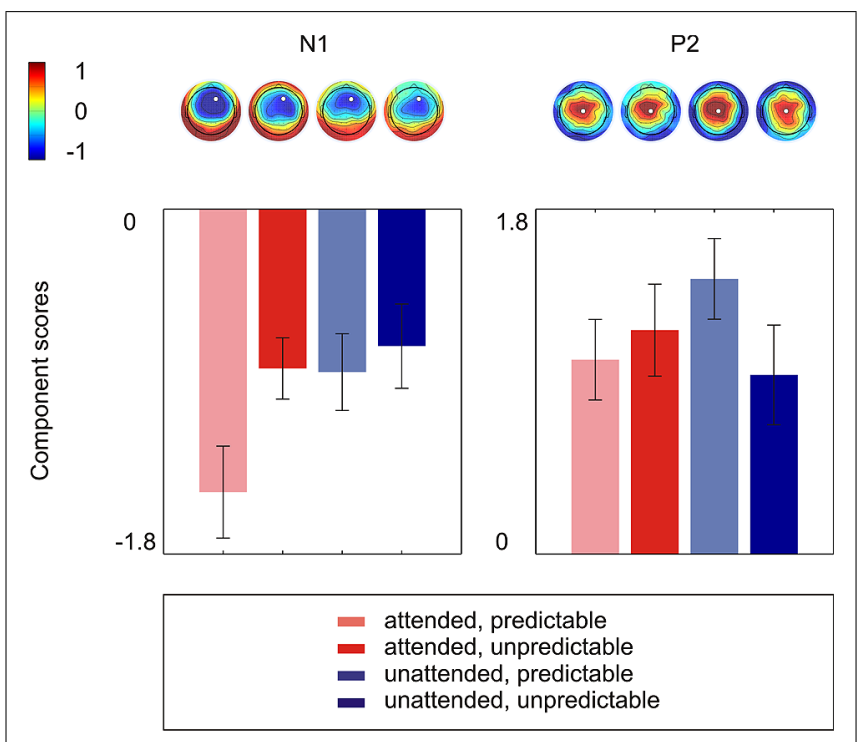

FIGURE 4 |The N1 and P2 component scores on electrodes showing the largest responses (N1: F2; P2: Cz) and the topographical distribution of components in each condition. Error bars depict one standard error above and below the mean.

localization (Scherg and von Cramon, 1986). A four-shell ellipsoidal head model was used. Two equivalent current dipoles were randomly seeded and freely fitted. The analysis showed that all four conditions had sources close to each other in the bilateral auditory areas (Figure 5). The average Talairach coordinates across the four conditions (left, $x$ : $-40.5, y:-20.9, z$ : 6.5 ; right, $x: 37.0, y:-18.9, z: 13.5)$ are close to the approximate location of the Heschl's gyrus (e.g., the average limits in Penhune etal., 1996 are for the left $x$ : -32.5 to $-62.3, y$ : -33.2 to $-4.9, z: 1.4$ to 14.2 and for the right $x: 34.7$ to 58.2, $y:-32.8$ to $-4.7, z$ : 1.1 to 17.7$)$. The two dipole model had residual variances of $1.22 \%$ (attended/predictable), $1.62 \%$ (attended/unpredictable), 2.51\% (unattended/predictable), and $2.45 \%$ (unattended/unpredictable). The result suggests that the effects of attention and prediction are based on the activation of neuronal populations in the bilateral auditory areas.

\section{P2}

In the $2 \times 2$ repeated measures ANOVA, neither the main effect of attention $[F(1,15)=0.44, p=0.52]$ nor the main effect of prediction $[F(1,15)=0.70, p=0.42]$ was significant. The attention $\times$ prediction interaction was marginally significant $[F(1,15)=4.15, p=0.06]$. However, there was no significant effect in Tukey LSD post hoc comparisons (Attention effect in predictable condition: $t(15)=-1.99, p=0.07$; Attention effect in unpredictable condition: $t(15)=1.08, p=0.30$; Prediction effect in attended condition: $t(15)=-0.61, p=0.55$; Prediction effect in unattended condition: $t(15)=1.84, p=0.09$ ).

\section{DISCUSSION}

The orthogonal design in the current study allowed us to evaluate whether attention and prediction are dependent of each other, and if so, how these two top-down mechanisms may interact for the optimization of perception. We found that attention and prediction interacted on the amplitude of the N1 ERP component generated in the auditory cortices. The N1 amplitude in the attended/predictable condition was larger than that in any of the other conditions. The relatively early latency of the interaction between these two variables is in line with the idea that attention and prediction can synergistically enhance perceptual analysis through top-down pathways (Doherty et al., 2005; Chaumon et al., 2008). Furthermore, it seems that attention and prediction rely on each other to form a tonically maintained set which can selectively include/exclude sensory input for further processing (Hillyard et al., 1973; Kok et al., 2012b). In other words, both attention and prediction are involved in the optimization of sensory processing.

Precious research reported that neurons in the sensory cortices can undergo short-term, task-dependent, and context-specific changes in the receptive field properties (Fritz et al., 2003, 2007, 2008). Such changes in the selectivity of sensory cortex neurons are believed to be the mechanism underlying the optimization of perception (Ahveninen et al., 2006; Kauramäki et al., 2007; Kok et al., 2012a). Extending the idea, our results further suggest

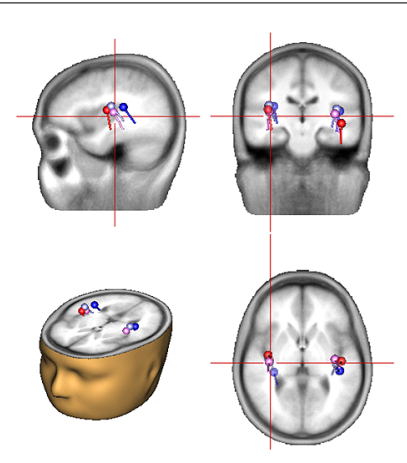

Right

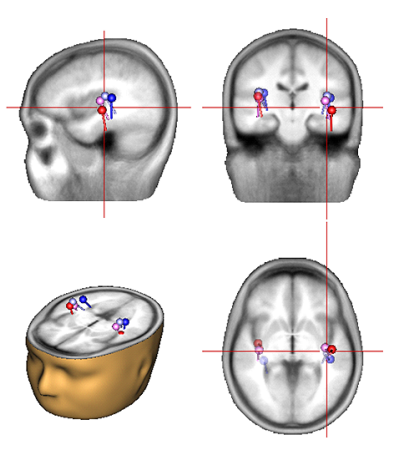

Left

FIGURE 5 | Source locations of the N1 response in each condition based on the topographical distribution of the component in the temporal PCA. 
that both attention and prediction are needed for such rapid plasticity to occur. Specifically, one may speculate that while attention determines the overall cortical responsiveness to stimuli (Lakatos et al., 2008; Busch etal., 2009; Schroeder and Lakatos, 2009), prediction serves as an anchor for the selective modulation of synaptic input strengths. An interesting question for future research is whether it is possible to disentangle the synergistic neuronal effects of attention and prediction on sensory processing.

The prediction-dependent attention effect demonstrates, for the first time, that attention alone may not be able to reshape perceptual inferences. It is unknown whether the prediction participants formed in the current study was implicit or explicit. However, it seems that prediction, be it implicit or explicit, is needed for attention effects to occur. At first glance, this idea seems difficult to reconcile with previous studies showing that attention can selectively modulate the response of neuronal subpopulations that prefer the attended stimulus features (Martinez-Trujillo and Treue, 2004; Liu et al., 2007). However, it is noteworthy that, in previous studies, attended stimulus features were also predictable stimulus features, making it difficult to disentangle the influence of prediction on attention effects. Our finding of the predictiondependent attention effect suggests that prediction may be an important construct for attention.

The attention-dependent prediction effect, on the other hand, sheds light on the dynamic influence of attention on prediction. Notably, using functional magnetic resonance imaging (fMRI) and visual stimuli, Kok etal. (2012b) reported an approach of manipulating attention and prediction that is similar to ours. In the attended condition, our finding of predictionrelated enhancement of the N1 amplitude from the auditory cortices was consistent with Kok et al.'s finding of predictionrelated enhancement of activation in early visual cortex. Such enhancement of neuronal responses confirm the notion that the engagement of attention may increase the weighting of sensory information, resulting in enhanced neuronal responses to predictable stimuli relative to unpredictable stimuli (Rao, 2005). The enhanced N1 is nicely in line with the predictive coding theory of sensory processing. The predictive coding theory suggests that perception entails the propagation of signals from two distinct types of neuronal populations, the representation neurons, and the prediction error neurons (Friston, 2005, 2009; Egner etal., 2010). Although there was some debate in recent neurocomputational research concerning which type of neurons is modulated by attention (Spratling, 2008, 2010; Feldman and Friston, 2010), it was shown that both models presume enhanced responses to predictable stimuli relative to unpredictable stimuli in the attended condition. In contrast, in the unattended condition, our null result was inconsistent with Kok et al.'s finding of prediction-related suppression of activation in early visual cortex. While caution should be taken for the interpretation of our null result, we speculate that the discrepancy may be related to the different manipulations of attention and prediction.

Concerning the manipulation of attention, the discrepancy may be explained by a competition hypothesis borrowed from research on mismatch negativity $(\mathrm{MMN})$. Mismatch negativity is generated to violations of abstract stimulus prediction rules (Tervaniemi etal., 1994; Paavilainen et al., 1999), reflecting the detection of deviant stimuli independent of attention (Näätänen, 1990; Näätänen et al., 2007). Importantly, a competition hypothesis suggests that, although attention does not affect the deviant detection process per se, it may influence the sensory information reaching the deviant detection process (Sussman et al., 2003). For example, when certain stimulus features are attended in a competing stimulus stream, the detection of stimuli deviating in certain stimulus features can be accordingly abolished in the unattended channel, leading to the absence of the MMN.

We suggest that whether the prediction effect appears in the unattended condition depends on the degree of such competition for cognitive resources. In the current study, attention was manipulated in the "filtering" manner. In this case, participants may inhibit the processing of unattended stimuli stream at an early stage to get rid of unnecessary information. In the study of Kok etal. (2012b), on the contrary, attention was manipulated in the "probabilistic" manner. In this case, participants may not inhibit the processing of unattended stimuli until the level concerning behavioral output. Consequently, the competition for cognitive recourses may be too weak to eliminate the possibility that unpredictable stimuli automatically attract attention, resulting in enhanced neuronal responses (Larsson and Smith, 2012). The same line of argumentation holds for previous studies demonstrating prediction-related suppression effects, in which participants' attention was either not controlled (Schafer and Marcus, 1973; Stefanics et al., 2010; Besle et al., 2011) or directed away from the manipulation of prediction using a cover task (Schafer et al., 1981; Summerfield et al., 2008; Lange, 2009; Todorovic etal., 2011; Kok etal., 2012a; Todorovic and de Lange, 2012). Given that participants' attention still stayed focused on the stimuli in these tasks, it is difficult to exclude the possibility that the observed prediction-related suppression effects reflected the enhanced neuronal responses to unpredictable stimuli.

Alternatively, whether the prediction effect appears in the unattended condition may depend on the particular manipulation of prediction. In the current study, the unpredictable condition consisted of stimuli randomly selected from a given frequency set. In other words, it is difficult for participants to form a specific prediction about the frequency of the upcoming stimuli in the first place. Therefore, the activation in sensory cortex may reflect exclusively the neuronal signals triggered by the presence of unpredictable stimuli. In the study of Kok et al. (2012b), on the contrary, the unpredictable condition consisted of stimuli which were presented as oddballs that violated participants' prediction. Consequently, the activation in sensory cortex may reflect not only the neuronal signals triggered by the presence of unpredictable stimuli but also the neuronal signals triggered by the absence of predictable stimuli. Such increase in the neuronal signals in the unpredictable condition may be the reason why prediction-related suppression can be observed.

On the other hand, no significant effect was found in the P2 time window. At best, there was a marginally significant interaction between attention and prediction. While caution should be taken in interpreting the results, the pattern of the interaction 
suggests that attention reversed the direction of prediction effects on the P2. In the attended condition, predictable stimuli suppressed the $\mathrm{P} 2$. In the unattended condition, predictable stimuli enhanced the P2. Interestingly, the pattern is opposite to the findings of Kok et al. (2012b), where prediction-related hemodynamic responses in early visual cortex were enhanced when the stimuli were attended and suppressed when the stimuli were unattended. More research is needed to examine whether the interaction between attention and prediction may change over time and how such dynamics manifest in hemodynamic responses.

Overall, our findings suggest that future research on attention and its relation to prediction needs to differentiate between different manipulations of attention and prediction. Moreover, our results confirm the importance of incorporating the modulatory effect of attention in the predictive coding theory. The predictive coding theory postulates that the prediction effect indexes the difference in neurocomputational demand for predictable/unpredictable information (Friston, 2005; Egner et al., 2010). Specifically, it is suggested that predictable information, relative to unpredictable information, elicits smaller prediction error signals. The smaller prediction error signals are associated with reduced connectivity within a hierarchical cortical network (Garrido et al., 2007, 2009), which accounts for the observations of prediction-related suppression in evoked responses (Wacongne et al., 2011). The predictive coding theory seems contradictory to our findings of significant prediction-related N1 enhancement in the attended condition and the lack of prediction-related N1 suppression in the unattended condition, unless the modulatory effect of attention is taken into consideration. Recent computational models demonstrated that attention emerges naturally in a similar Bayes-optimal scheme as the inference about the precision of the causes of sensory input (Friston, 2009; Feldman and Friston, 2010). It is, thus, possible that the prediction effect does not reflect merely the different prediction error signals. Rather, it reflects how the precision of the causes of sensory input is weighed against the prediction error signals. In other words, it is the attention effect, rather than the prediction effect, that is dominant here. In the attended condition, the precision of the causes of sensory input increases. The increased precision may manifest as an imaginary tuning curve with narrower bandwidth. Moreover, it may outweigh the prediction error signals to predictable input over the prediction error signals to unpredictable input. This is when the prediction-related enhancement can be observed. In the unattended condition, on the other hand, the precision of the causes of sensory input decreases. It can manifest as an imaginary tuning curve with wider bandwidth. In this case, the amount of the decrease in precision may determine to what extent the prediction error signals to unpredictable input can be canceled out. The larger the decrease in precision, the more it cancels out the prediction error signals to unpredictable input. At some point, the prediction error signals to unpredictable input would become really small, rendering the prediction-related suppression negligible. A real life analogy is that, when one pays minimum amount of attention to the stimuli, the boundary between the unpredictable input and the predictable input is of little importance. This mechanism could be tested in detail in future research.

\section{ACKNOWLEDGMENTS}

This research was supported by a grant from the Agence Nationale de la Recherche (INTACT ANR-09-BLAN-0318). We thank Trevor Agus for help on stimulus calibration and the Paris Descartes Platform for Sensorimotor Studies (Université Paris Descartes, CNRS, INSERM, Région Ile-de-France) for supporting the experimental work presented here.

\section{REFERENCES}

Ahveninen, J., Jääskeläinen, I. P., Raij, T., Bonmassar, G., Devore, S., Hämäläinen, M., etal. (2006). Task-modulated "what" and "where" pathways in human auditory cortex. Proc. Natl. Acad. Sci. U.S.A. 103, 14608-14613. doi: 10.1073/pnas.0510480103

Alcaini, M., Giard, M. H., Thévenet, M., and Pernier, J. (1994). Two separate frontal components in the N1 wave of the human auditory evoked response. Psychophysiology 31, 611-615. doi: 10.1111/j.1469-8986.1994.tb02354.x

Besle, J., Schevon, C. A., Mehta, A. D., Lakatos, P., Goodman, R. R., McKhann, G. M., et al. (2011). Tuning of the human neocortex to the temporal dynamics of attended events. J. Neurosci. 31, 3176-3185. doi: 10.1523/JNEUROSCI.451810.2011

Busch, N. A., Dubois, J., and VanRullen R. (2009). The phase of ongoing EEG oscillations predicts visual perception. J. Neurosci. 29, 7869-7876. doi: 10.1523/JNEUROSCI.0113-09.2009

Chaumon, M., Drouet, V., and Tallon-Baudry, C. (2008). Unconscious associative memory affects visual processing before $100 \mathrm{~ms}$. J. Vis. 8, 1-10. doi: 10.1167/8.3.10

Chawla, D., Rees, G., and Friston, K. J. (1999). The physiological basis of attentional modulation in extrastriate visual areas. Nat. Neurosci. 2, 671-676. doi: $10.1038 / 10230$

Corbetta, M., and Shulman, G. L. (2002). Control of goal-directed and stimulusdriven attention in the brain. Nat. Rev. Neurosci. 3, 201-215. doi: 10.1038/nrn755 Costa-Faidella, J., Baldeweg, T., Grimm, S., and Escera, C. (2011). Interactions between "what" and "when" in the auditory system: temporal predictability enhances repetition suppression. J. Neurosci. 31, 18590-18597. doi: 10.1523/JNEUROSCI.2599-11.2011

Delorme, A., and Makeig, S. (2004). EEGLAB: an open source toolbox for analysis of single-trial EEG dynamics including independent component analysis. J. Neurosci. Methods 134, 9-21. doi: 10.1016/j.jneumeth.2003.10.009

Dien, J. (2012). Applying principal components analysis to event-related potentials: a tutorial. Dev. Neuropsychol. 37, 497-517. doi: 10.1080/87565641.2012.697503

Dien, J., Beal, D. J., and Berg, P. (2005). Optimizing principal components analysis of event-related potentials: matrix type, factor loading weighting, extraction, and rotations. Clin. Neurophysiol. 116, 1808-1825. doi: 10.1016/j.clinph.2004.11.025

Doherty, J. R., Rao, A., Mesulam, M. M., and Nobre, A. C. (2005). Synergistic effect of combined temporal and spatial expectations on visual attention. J. Neurosci. 25, 8259-8266. doi: 10.1523/JNEUROSCI.1821-05.2005

Egner, T., Monti, J. M., and Summerfield, C. (2010). Expectation and surprise determine neural population responses in the ventral visual stream. J. Neurosci. 30, 16601-16608. doi: 10.1523/JNEUROSCI.2770-10.2010

Evans, K. M., and Federmeier, K. D. (2007). The memory that's right and the memory that's left: event-related potentials reveal hemispheric asymmetries in the encoding and retention of verbal information. Neuropsychologia 45, 1777-1790. doi: 10.1016/j.neuropsychologia.2006.12.014

Feldman, H., and Friston, K. J. (2010). Attention, uncertainty, and free-energy. Front. Hum. Neurosci. 4:215. doi: 10.3389/fnhum.2010.00215

Friston, K. J. (2005). A theory of cortical responses. Philos. Trans. R. Soc. B Biol. Sci. 360, 815-836. doi: 10.1098/rstb.2005.1622

Friston, K. J. (2009). The free-energy principle: a rough guide to the brain? Trends Cogn. Sci. 13, 293-301. doi: 10.1016/j.tics.2009.04.005

Fritz, J., Elhilali, M., David, S., and Shamma, S. (2007). Auditory attention focusing the searchlight on sound. Curr. Opin. Neurobiol. 17, 437-455. doi: 10.1016/j.conb.2007.07.011

Fritz, J., Elhilali, M., David, S., and Shamma, S. (2008). Does attention play a role in dynamic receptive field adaptation to changing acoustic salience in A1? Hear. Res. 229, 186-203. doi: 10.1016/j.heares.2007.01.009

Fritz, J., Shamma, S., Elhilali, M., and Klein, D. (2003). Rapid task-related plasticity of spectrotemporal receptive fields in primary auditory cortex. Nat. Neurosci. 6, 1216-1223. doi: 10.1038/nn1141 
Garrido, M. I., Kilner, J. M., Kiebel, S. J., and Friston, K. J. (2007). Evoked brain responses are generated by feedback loops. Proc. Natl. Acad. Sci. U.S.A. 104, 20961-20966. doi: 10.1073/pnas.0706274105

Garrido, M. I., Kilner, J. M., Kiebel, S. J., Stephan, K. E., Baldeweg, T., and Friston, K. J. (2009). Repetition suppression and plasticity in the human brain. Neuroimage 48, 269-279. doi: 10.1016/j.neuroimage.2009.06.034

Hillyard, S. A., Hink, R. F., Schwent, V. L., and Pieton, T. W. (1973). Electrical signs of selective attention in the human brain. Science 182, 177-180. doi 10.1126/science.182.4108.177

Jääskeläinen, I. P., Ahveninen, J., Belliveau, J. W., Raij, T., and Sams, M. (2007) Short-term plasticity in auditory cognition. Trends Neurosci. 30, 653-661. doi: 10.1016/j.tins.2007.09.003

Kastner, S., Pinsk, M. A., de Weerd, P., Desimone, R., and Ungerleider, L. G. (1999). Increased activity in human visual cortex during directed attention in the absence of visual stimulation. Neuron 22, 751-761. doi: 10.1016/S0896-6273(00)80734-5

Kauramäki, J., Jääskeläinen, I. P., and Sams, M. (2007). Selective attention increases both gain and feature selectivity of the human auditory cortex. PLoS ONE 2:e909. doi: 10.1371/journal.pone.0000909

Kayser, J., and Tenke, C. E. (2003). Optimizing PCA methodology for ERP component identification and measurement: theoretical rationale and empirical evaluation. Clin. Neurophysiol. 114, 2307-2325. doi: 10.1016/S1388-2457(03)00241-4

Kayser, J., and Tenke, C. E. (2006). Consensus on PCA for ERP data, and sensibility of unrestricted solutions. Clin. Neurophysiol. 117, 703-707. doi: 10.1016/j.clinph.2005.11.015

Kok, P., Jehee, J. F. M., and de Lange, F. P. (2012a). Less is more: expectation sharpens representations in the primary visual cortex. Neuron $75,265-270$. doi 10.1016/j.neuron.2012.04.034

Kok, P., Rahnev, D., Jehee, J. F. M., Lau, H. C., and de Lange, F. P. (2012b). Attention reverses the effect of prediction in silencing sensory signals. Cereb. Cortex 22, 2197-2206. doi: 10.1093/cercor/bhr310

Lakatos, P., Karmos, G., Mehta, A. D., Ulbert, I., and Schroeder, C. E. (2008) Entrainment of neuronal oscillations as a mechanism of attentional selection. Science 320, 110-113. doi: 10.1126/science.1154735

Lange, K. (2009). Brain correlates of early auditory processing are attenuated by expectations for time and pitch. Brain Cogn. 69, 127-137. doi: 10.1016/j.bandc.2008.06.004

Lange, K. (2013). The ups and downs of temporal orienting: a review of auditory temporal orienting studies and a model associating the heterogeneous findings on the auditory N1 with opposite effects of attention and prediction. Front. Hum. Neurosci. 7:263. doi: 10.3389/fnhum.2013.00263

Lange, K., Krämer, U. M., and Röder, B. (2006). Attending points in time and space. Exp. Brain Res. 173, 130-140. doi: 10.1007/s00221-006-0372-3

Lange, K., and Röder, B. (2006). Orienting attention to points in time improves stimulus processing both within and across modalities. J. Cogn. Neurosci. 18 715-729. doi: 10.1162/jocn.2006.18.5.715

Lange, K., Rösler, F., and Röder, B. (2003). Early processing stages are modulated when auditory stimuli are presented at an attended moment in time: an event-related potential study. Psychophysiology 40, 806-817. doi: 10.1111/14698986.00081

Larsson, J., and Smith, A. T. (2012). fMRI repetition suppression: neuronal adaptation or stimulus expectation? Cereb. Cortex 22, 567-576. doi: 10.1093/cercor/bhr119

Liu, T., Larsson, J., and Carrasco, M. (2007). Feature-based attention modulates orientation-selective responses in human visual cortex. Neuron 55, 313-323. doi: 10.1016/j.neuron.2007.06.030

Luck, S. J. (2005). An Introduction to the Event-Related Potential Technique. Cambridge: The MIT Press.

Martinez-Trujillo, J. C., and Treue, S. (2004). Feature-based attention increases the selectivity of population responses in primate visual cortex. Curr. Biol. 14, 744-751. doi: 10.1016/j.cub.2004.04.028

Melloni, L., Schwiedrzik, C. M., Müller, N., Rodriguez, E., and Singer, W. (2011). Expectations change the signatures and timing of electrophysiological correlates of perceptual awareness. J. Neurosci. 31, 1386-1396. doi: 10.1523/JNEUROSCI.4570-10.2011

Näätänen, R. (1990). The role of attention in auditory information processing as revealed by event-related potentials and other brain measures of cognitive function. Behav. Brain Sci. 13, 201-288. doi: 10.1017/S0140525X000 78407
Näätänen, R., Paavilainen, P., Rinne, T., and Alho, K. (2007). The mismatch negativity (MMN) in basic research of central auditory processing: a review. Clin. Neurophysiol. 118, 2544-2590. doi: 10.1016/j.clinph.2007. 04.026

Paavilainen, P., Jaramillo, M., Näätänen, R., and Winkler, I. (1999). Neuronal populations in the human brain extracting invariant relationships from acoustic variance. Neurosci. Lett. 265, 179-182. doi: 10.1016/S0304-3940(99) 00237-2

Penhune, V. B., Zatorre, R. J., MacDonald, J. D., and Evanc, A. C. (1996). Interhemispheric anatomical differences in human primary auditory cortex: probabilistic mapping and volume measurement from magnetic resnonance scans. Cereb. Cortex 6, 661-672. doi: 10.1093/cercor/6.5.661

Rao, R. P. N. (2005). Bayesian inference and attentional modulation in the visual cortex. Neuroreport 16, 1843-1848. doi: 10.1097/01.wnr.0000183900.92901.fc

Rohenkohl, G., Cravo, A. M., Wyart, V., and Nobre, A. C. (2012). Temporal expectation improves the quality of sensory information. J. Neurosci. 32, 8424-8428. doi: 10.1523/JNEUROSCI.0804-12.2012

SanMiguel, I., Todd, J., and Schröger, E. (2013). Sensory suppression effects to self-initiated sounds reflect the attenuation of the unspecific N1 component of the auditory ERP. Psychophysiology 50, 334-343. doi: 10.1111/psyp. 12024

Schafer, E. W., Amochaev, A., and Russell, M. J. (1981). Knowledge of stimulus timing attenuates human evoked cortical potentials. Electroencephalogr. Clin. Neurophysiol. 52, 9-17. doi: 10.1016/0013-4694(81) 90183-8

Schafer, E. W., and Marcus, M. M. (1973). Self-stimulation alters human sensory brain responses. Science 181, 175-177. doi: 10.1126/science.181.4095.175

Scherg, M., and von Cramon, D. (1986). Evoked dipole source potentials of the human auditory cortex. Electroencephalogr. Clin. Neurophysiol. 65, 344-360. doi: 10.1016/0168-5597(86)90014-6

Schroeder, C. E., and Lakatos, P. (2009). Low-frequency neuronal oscillations as instruments of sensory selection. Trends Neurosci. 32, 9-18. doi: 10.1016/j.tins.2008.09.012

Spratling, M. W. (2008). Reconciling predictive coding and biased competition models of cortical function. Front. Comput. Neurosci. 2:4. doi: 10.3389/neuro.10.004.2008

Spratling, M. W. (2010). Predictive coding as a model of response properties in cortical area V1. J. Neurosci. 30, 3531-3543. doi: 10.1523/JNEUROSCI.491109.2010

Stefanics, G., Hangya, B., Hernádi, I., Winkler, I., Lakatos, P., and Ulbert, I. (2010). Phase entrainment of human delta oscillations can mediate the effects of expectation on reaction speed. J. Neurosci. 30, 13578-13585. doi: 10.1523/JNEUROSCI.0703-10.2010

Summerfield, C., and Egner, T. (2009). Expectation (and attention) in visual cognition. Trends Cogn. Sci. 13, 403-409. doi: 10.1016/j.tics.2009.06.003

Summerfield, C., and Egner, T. (2013). "Attention and decision-making," in The Oxford Handbook of Attention, eds A. C. Nobre and S. Kastner (Oxford: Oxford University Press).

Summerfield, C., Trittschuh, E. H., Monti, J. M., Mesulam, M. M., and Egner, T. (2008). Neural repetition suppression reflects fulfilled perceptual expectations. Nat. Neurosci. 11, 1004-1006. doi: 10.1038/nn.2163

Sussman, E., Winkler, I., and Wang, W. (2003). MMN and attention: competition for deviance detection. Psychophysiology 40, 430-435. doi: 10.1111/1469-8986. 00045

Tervaniemi, M., Saarinen, J., Paavilainen, P., Danilova, N., and Näätänen, R. (1994). Temporal integration of auditory information in sensory memory as reflected by the mismatch negativity. Biol. Psychol. 38, 157-167. doi: 10.1016/0301-0511(94)90036-1

Timm, J., SanMiguel, I., Saupe, K., and Schröger, E. (2013). The N1-suppression effect for self-initiated sounds is independent of attention. BMC Neurosci. 14:2. doi: 10.1186/1471-2202-14-2

Todorovic, A., and de Lange, F. P. (2012). Repetition suppression and expectation suppression are dissociable in time in early auditory evoked fields. J. Neurosci. 32, 13389-13395. doi: 10.1523/JNEUROSCI.2227-12.2012

Todorovic, A., van Ede, F., Maris, E., and de Lange, F. P. (2011). Prior expectation mediates neural adaptation to repeated sounds in the auditory cortex an MEG Study. J. Neurosci. 31, 9118-9123. doi: 10.1523/JNEUROSCI.1425-11. 2011 
Wacongne, C., Labyt, E., van Wassenhove, V., Bekinschtein, T., Naccache, L., and Dehaene, S. (2011). Evidence for a hierarchy of predictions and prediction errors in human cortex. Proc. Natl. Acad. Sci. U.S.A. 108, 20754-20759. doi: $10.1073 /$ pnas. 1117807108

Conflict of Interest Statement: The authors declare that the research was conducted in the absence of any commercial or financial relationships that could be construed as a potential conflict of interest.

Received: 22 January 2014; accepted: 28 February 2014; published online: 26 March 2014
Citation: Hsu Y-F, Hämäläinen JA and Waszak F (2014) Both attention and prediction are necessary for adaptive neuronal tuning in sensory processing. Front. Hum. Neurosci. 8:152. doi: 10.3389/fnhum.2014.00152

This article was submitted to the journal Frontiers in Human Neuroscience.

Copyright (c) 2014 Hsu, Hämäläinen and Waszak. This is an open-access article distributed under the terms of the Creative Commons Attribution License (CC BY). The use, distribution or reproduction in other forums is permitted, provided the original author(s) or licensor are credited and that the original publication in this journal is cited, in accordance with accepted academic practice. No use, distribution or reproduction is permitted which does not comply with these terms. 